\title{
Hierarchical iridium-based multimetallic alloy with double-core-shell architecture for efficient overall water splitting
}

\author{
Jie Zhang ${ }^{1}$, Zelin Chen ${ }^{1}$, Chang Liu ${ }^{1}$, Jun Zhao ${ }^{1}$, Siliang Liu ${ }^{1}$, Dewei Rao ${ }^{2}$, Anmin Nie ${ }^{3}$, \\ Yanan Chen $^{1^{*}}$, Yida Deng ${ }^{1 *}$ and Wenbin $\mathrm{Hu}^{1,4}$
}

\begin{abstract}
The overall water splitting for hydrogen production is an effective strategy to resolve the environmental and energy crisis. Here, we report a facile approach to synthesize the Ir-based multimetallic, hierarchical, double-coreshelled architecture (HCSA) assisted by oil bath reaction for boosting overall water splitting in acidic environment. The IrNiCu HCSA shows superior electrocatalytic activity for hydrogen evolution reaction (HER) and oxygen evolution reaction (OER), which are comparable to commercial $\mathrm{Pt} / \mathrm{C}$ and better than $\mathrm{IrO}_{2}$. The IrNiCu HCSA exhibits remarkably catalytic efficiency as bifunctional catalyst for overall water splitting where a low cell voltage of $1.53 \mathrm{~V}$ is enough to drive a

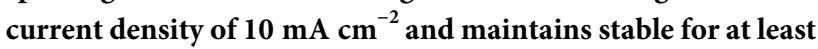
$20 \mathrm{~h}$. The presented work for the design and synthesis of novel Ir-based multimetallic architecture paves the way for highperformance overall water splitting catalysis.
\end{abstract}

Keywords: IrNiCu nanoframe, hierarchical structure, core-shell, oxygen evolution reaction, hydrogen evolution reaction

\section{INTRODUCTION}

Electrochemical water splitting is considered as one of the most promising way to produce hydrogen [1-3], which composes of hydrogen evolution reaction (HER) of cathodic half-reaction and oxygen evolution reaction (OER) of anodic half-reaction [4-11]. Acid polymer electrolyte membrane water electrolyzer (PEMWE) is the most popular technique towards the production of hydrogen and oxygen fuels [12-17]. Pt and Ir/Ru-based nanocatalysts are emerging as the state-of-the-art metal catalysts for HER and OER in PEMWE, respectively [2,18-22]. However, these catalysts feature moderate intrinsic electrocatalytic activity with sluggish kinetics of HER and OER, and low durability in harsh acidic media and high cost, discouraging their large-scale applications [23-25]. Therefore, numerous scientific research efforts have been devoted to investigating and designing efficient noble metal nanocatalysts with high electrocatalytic activity, long-term stability and the use of non-noble metals. Alloying Ir with transition group metals [19,24-34] is generally regarded as an effective strategy that not only substantially reduces the usage of Ir but also facilitates the catalytic activity and stability for water splitting by tuning the d-band structure and oxygen adsorption energy on the surface of Ir. Another strategy to optimize the overall catalytic activity is to tune the geometric shape of the nanocatalysts [18,35-42]. A hierarchical alloy architecture is believed to show the capability of promoting the initial activity with more active surface area exposed to reactants [43]. The construction of a core-shell structure with a noble metal shell also demonstrates the enhancement of catalytic activity and stability, the core-shell structure maximizes valuable metal exposure to reactants and minimizes the amount of expensive metal used, and it boosts catalysis via core-shell synergistic interactions, such as tuning the electronic and surface strain effects $[44,45]$. However, it is still a huge challenge to synthesize a three-dimensional (3D), hierarchical, core-shell struc-

\footnotetext{
${ }^{1}$ School of Materials Science and Engineering, Key Laboratory of Advanced Ceramics and Machining Technology of Ministry of Education, Tianjin Key Laboratory of Composite and Functional Materials, Tianjin University, Tianjin 300072, China

${ }^{2}$ School of Materials Science and Engineering, Jiangsu University, Zhenjiang 212013, China

${ }^{3}$ State Key Lab of Metastable Materials Science and Technology, Yanshan University, Qinhuangdao 066004, China

${ }^{4}$ Joint School of National University of Singapore and Tianjin University, Internatioal Campus of Tianjin University, Fuzhou 350207, China

* Corresponding authors (emails: yida.deng@tju.edu.cn (Deng Y); yananchen@tju.edu.cn (Chen Y))
} 
tured Ir-based multimetallic alloy with improved intrinsic activity and increased active sites as a highly efficient and stable bifunctional catalyst for both HER and OER.

In this work, we report a facile, one-pot strategy for a controllable synthesis of 3D hierarchical Ir-based multimetallic alloy nanoframes assembled by $\mathrm{IrNiCu}$ nanocrystals, with a Cu-rich@Ni-rich@Ir-rich multielement alloy and double-core-shell architecture (Fig. 1), as highly active and exceptionally stable bifunctional electrocatalysts for overall water splitting. The as-synthesized $\mathrm{IrNiCu}$ hierarchical double-core-shelled architecture (HCSA) exhibits remarkable electrocatalytic performance for both HER and OER in acidic environments. Specifically, the IrNiCu HCSA shows outstanding HER activity with a low overpotential of $41 \mathrm{mV}$ at a current density of $10 \mathrm{~mA} \mathrm{~cm}^{-2}$ and a small Tafel slope of $21.4 \mathrm{mV} / \mathrm{dec}$, which is comparable to commercial platinum/carbon (Pt/ C) electrocatalysts $(39 \mathrm{mV}$ and $21.0 \mathrm{mV} / \mathrm{dec})$. The $\mathrm{IrNiCu}$ HCSA displays the highest electrocatalytic activity for OER with only a $262 \mathrm{mV}$ overpotential to attain $10 \mathrm{~mA} \mathrm{~cm}^{-2}$ and a $71.4 \mathrm{mV} / \mathrm{dec}$ Tafel slope, which outperforms the commercial $\mathrm{IrO}_{2} \quad(326 \mathrm{mV}$ and $77.3 \mathrm{mV} / \mathrm{dec}$ ), indicating that the $\mathrm{IrNiCu} \mathrm{HCSA}$ is an excellent OER electrocatalyst in acidic electrolyte. It is noteworthy that the IrNiCu HCSA represents impressive electrocatalytic activity and superior durability for overall water splitting, achieving a $10 \mathrm{~mA} \mathrm{~cm}^{-2}$ current at a low cell voltage of $1.53 \mathrm{~V}$ and sustaining a negligible decay of polarized current after 1000 cycles. The present work provides possibilities in tuning the hierarchical and coreshell structure of Ir-based multimetallic nanocatalysts for promoting the electrocatalytic performance for overall water splitting.

\section{EXPERIMENTAL SECTION}

\section{Chemicals}

Iridium(III) 2,4-pentanedionate ( $\left.\operatorname{Ir}(\mathrm{acac})_{3}, \operatorname{Ir} 37.5 \%\right)$, nickel(II) 2,4-pentanedionate ( $\left.\mathrm{Ni}(\mathrm{acac})_{2}, 96 \%\right)$, copper(II) acetylacetonate $\left(\mathrm{Cu}(\mathrm{acac})_{2}, 98 \%\right)$, (1-hexadecyl)trimethylammonium chloride (CTAC, 96\%), and 1,2-hexadecanediol (1,2-HDD, 98\%) were purchased from Alfa Aesar. Oleylamine (80\%-90\%) was manufactured by Sigma-Aldrich Corporation. All the chemicals were used as purchased without further purification and deionized water was employed in these experiments.

\section{Synthesis of the IrNiCu HCSA}

The IrNiCu nanoframe structures were one-step synthesized with an oil bath method. A suspension containing $26.1 \mathrm{mg} \operatorname{Ir}(\mathrm{acac})_{3}, 10.7 \mathrm{mg} \mathrm{Ni}(\mathrm{acac})_{2}, 5.3 \mathrm{mg} \mathrm{Cu}(\mathrm{acac})_{2}$, $33.3 \mathrm{mg}$ CTAC, $10.5 \mathrm{mg}$ 1,2-HDD and $5 \mathrm{~mL}$ oleylamine was homogeneously dissolved with magnetic stirring at $60^{\circ} \mathrm{C}$ for $10 \mathrm{~min}$ in a round-bottom flask. After that, the round-bottom flask was transferred to a preheated silicone oil bath at $260^{\circ} \mathrm{C}$ for $40 \mathrm{~min}$ and then cooled to room temperature. The reacted mixture was collected by centrifugation with $5 \mathrm{~mL}$ methylbenzene and $10 \mathrm{~mL}$ methanol at 8000 revolutions per minute for $5 \mathrm{~min}$. To further purify the precipitate, the centrifugation was performed twice more with $5 \mathrm{~mL}$ methylbenzene and $10 \mathrm{~mL}$ ethanol at 8000 revolutions per minute for $5 \mathrm{~min}$. Finally, the purified $\mathrm{IrNiCu}$ nanoframe catalyst powder was redispersed in $1 \mathrm{~mL}$ ethanol for further use.

\section{Synthesis of the IrNi and IrCu nanoarchitectures (NA)}

The $\mathrm{IrNi}$ and $\mathrm{IrCu}$ were prepared and purified in the

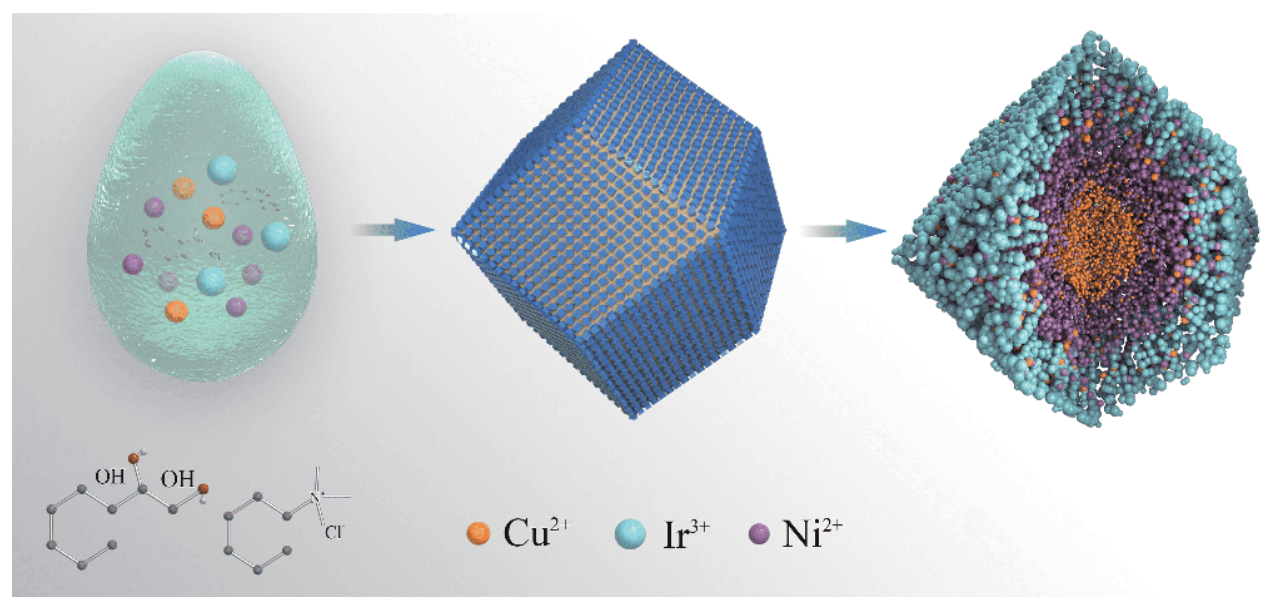

Figure 1 Schematic illustration of the synthesis of IrNiCu HCSA. 
same way, with $\operatorname{Ir}(\mathrm{acac})_{3}, \mathrm{Ni}(\mathrm{acac})_{2} / \mathrm{Cu}(\mathrm{acac})_{2}, \mathrm{CTAC}$, 1,2-HDD and oleylamine as the reactants. Finally, the purified $\mathrm{IrNi}$ or $\mathrm{IrCu}$ nanocatalyst powder was redispersed in $1 \mathrm{~mL}$ ethanol for further use.

\section{Characterizations}

The crystal structures of the as-prepared Ir-based catalysts were characterized by powder X-ray diffraction (XRD) conducted on a Bruker D8 Advance X-ray diffractometer $(\mathrm{Cu} \mathrm{Ka}$ radiation, $40 \mathrm{kV}, 40 \mathrm{~mA})$. Transmittance electron microscope (TEM) and high resolution TEM (HRTEM, JEOL JEM-2100F, $200 \mathrm{kV}$ and JEMARM200F) were used to study the particle morphologies. The surface chemical valence state was characterized using X-ray photoelectron spectroscopy (XPS, Thermo ESCALAB 250XI, with an incident radiation of monochromatic Al KR X-rays). The element contents of the asprepared nanoparticles were analyzed by inductively coupled plasma mass spectrometry (ICP-MS, Agilent 7700x).

\section{Electrochemical tests}

All electrochemical measurements were conducted on a CHI 660E electrochemical workstation (Shanghai Chenhua, China). The catalytic performance of HER and OER was carried out in a three-electrode system containing $0.5 \mathrm{~mol} \mathrm{~L}^{-1} \mathrm{H}_{2} \mathrm{SO}_{4}$ solution at room temperature. A glassy carbon electrode with diameter of $5 \mathrm{~mm}$, a graphite rod, and a $\mathrm{Ag} / \mathrm{AgCl}$ electrode $\left(3 \mathrm{~mol} \mathrm{~L}^{-1} \mathrm{KCl}\right)$ were serviced as the working, counter, and reference electrodes, respectively. Before every measurement, the glassy carbon electrode was polished with a $1.0 \mu \mathrm{m}$ alumina suspension on a piece of moist chamois and then repolished with a $0.3 \mu \mathrm{m}$ alumina suspension. Then, $8 \mu \mathrm{L}$ of the aforementioned $1 \mathrm{~mL}$ catalyst-ethanol ink was dropped twice on the working electrode with a pipette gun and left alone until the liquid had evaporated and then $8 \mu \mathrm{L}$ of a $0.2 \mathrm{wt} \%$ Nafion solution was droped. The loading of Ir on the glassy carbon electrode was $10 \mu \mathrm{g} \mathrm{cm}^{-2}$. The electrochemical potentials determined against the $\mathrm{Ag} / \mathrm{AgCl}$ reference electrode were converted to a reversible hydrogen electrode (RHE) scale via the Nernst equation: $E_{\mathrm{RHE}}=$ $E_{\mathrm{Ag} / \mathrm{AgCl}}+0.059 \mathrm{pH}+0.197$, and the overpotential $(\eta)$ for OER was calculated using the following equation: $\eta=$ $E_{\text {RHE }}-1.23 \mathrm{~V}$ [25].

Cyclic voltammograms (CVs) were performed to activate the active substances on the working electrodes before the linear sweep voltammetry (LSV), which was conducted to obtain polarization curves of the aforementioned samples. For HER tests, the CVs were re- corded in a $\mathrm{N}_{2}$-saturated solution in the potential range of 0 to $-0.4 \mathrm{~V}$ with scan rates of 100 and $20 \mathrm{mV} \mathrm{s}^{-1}$ for four cycles. Then, the LSVs were subsequently performed in the same potential range with a scan rate of $5 \mathrm{mV} \mathrm{s}^{-1}$ without $i R$ compensation [28]. For the OER activity, the CVs were first recorded in a $\mathrm{N}_{2}$-saturated solution in the potential range of 1.0 to $1.4 \mathrm{~V}$ at scan rates of 100 and $20 \mathrm{mV} \mathrm{s}^{-1}$ for four cycles. Then, the LSVs were subsequently performed in the same potential range with a scan rate of $5 \mathrm{mV} \mathrm{s}^{-1}$ without $i R$ compensation. Electrochemical impedance spectroscopy (EIS) was conducted from $100 \mathrm{kHz}$ to $0.01 \mathrm{~Hz}$ with an alternating current (AC) amplitude of $10 \mathrm{mV}$ at $-0.05 \mathrm{~V}$ versus $\mathrm{RHE}$ for HER and $1.5 \mathrm{~V}$ versus RHE for OER. Chronopotentiometry was employed to evaluate the stability of the as-prepared catalysts at a constant current density of $10 \mathrm{~mA} \mathrm{~cm}^{-2}$. To estimate the electrochemical active surface areas (ECSAs), the electrochemical double-layer capacitance $\left(C_{\mathrm{dl}}\right)$ was determined by CVs tested at different scan rates $(5,10$, $20,40,60,80$, and $100 \mathrm{mV} \mathrm{s}^{-1}$ ) in the potential windows of 0.9 to $1.0 \mathrm{~V}$ versus RHE [46].

For the overall water splitting, a two-electrode system with $0.5 \mathrm{~mol} \mathrm{~L}^{-1} \mathrm{H}_{2} \mathrm{SO}_{4}$ solution was used, and two carbon papers with a size of $1 \mathrm{~cm} \times 1 \mathrm{~cm}$ were used as the cathode and anode for HER and OER, respectively. The exact loading of the catalysts on the carbon papers was determined by ICP-MS. Polarization curves were carried out in the range of 1 to $2 \mathrm{~V}$ with a scan rate of $5 \mathrm{mV} \mathrm{s}^{-1}$. The durability tests of the overall water splitting were recorded using chronopotentiometry at a constant current density of $10 \mathrm{~mA} \mathrm{~cm}^{-2}$ for $20 \mathrm{~h}$.

\section{RESULTS AND DISCUSSION}

The morphology of IrNiCu HCSA synthesized by the one-step oil bath method was characterized by TEM. As shown in Fig. 2a, b and Fig. S1, the typical IrNiCu HCSAs were $3 \mathrm{D}$ polyhedron nanoframes composed of $\mathrm{IrNiCu}$ nanocrystals with diameters of $40-80 \mathrm{~nm}$. The HRTEM image of the $\mathrm{IrNiCu} \mathrm{HCSA}$ (Fig. 2c) reveals that the lattice spacing is $0.221 \mathrm{~nm}$, between $0.222 \mathrm{~nm}$ ( Ir, JCPDS card No. 06-0598), $0.203 \mathrm{~nm}$ (Ni, JCPDS card No. 040850), and $0.209 \mathrm{~nm}$ (Cu, JCPDS card No. 04-0836), indicating the formation of the (111) plane of $\mathrm{IrNiCu}$ [18]. High-angle annular dark field scanning tunneling electron microscopy (HAADF-STEM) and energy dispersive $\mathrm{X}$-ray spectroscopy (EDX) (Fig. 2d, e) were utilized to probe the elemental mapping of the $\mathrm{IrNiCu} \mathrm{HCSA}$ structure. From the higher magnification of the HAADFSTEM images, the iridium, nickel, and copper elements are distributed in a specific manner. The copper is most 

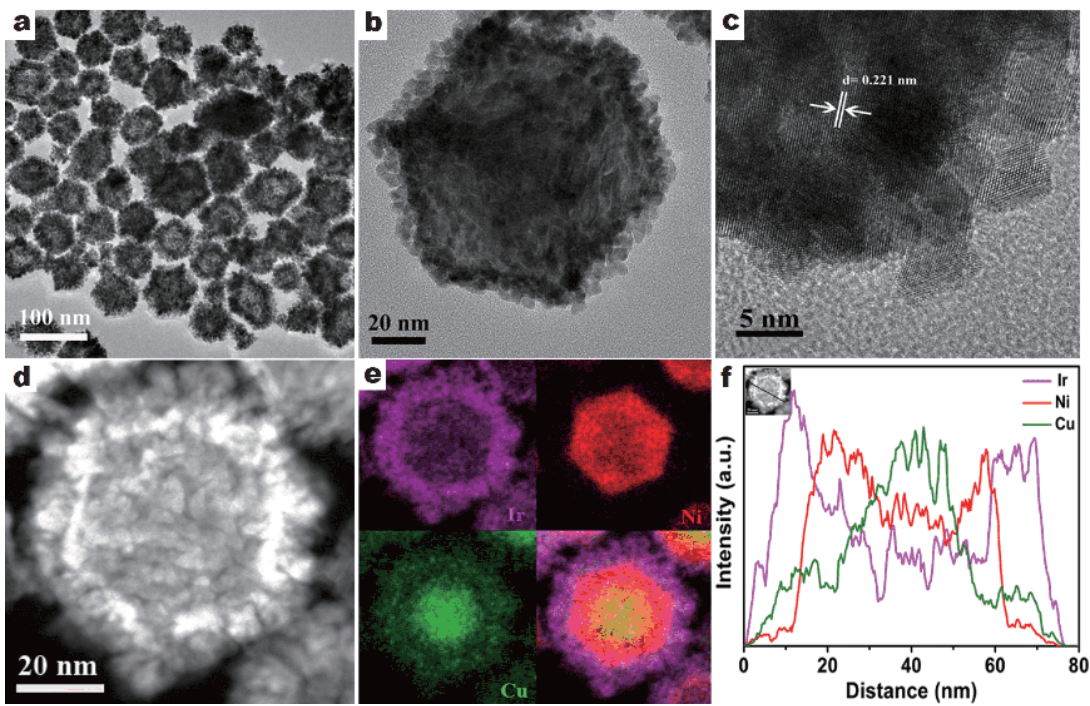

Figure 2 (a) TEM, (b) high-magnification TEM, (c) HRTEM, (d, e) HAADF-STEM image and corresponding EDX elemental mapping, and (f) linear scan element distribution of the as-synthesized IrNiCu HCSA.

concentrated in the middle, suggesting a preferential nucleation of copper during the synthesis process as the center of the whole structure. Then, nickel coats on the copper-rich phase and forms a certain orientation. Finally, iridium grows at the outermost layers of the $\mathrm{CuNi}$ rich $3 \mathrm{D}$ polyhedron. On the whole, iridium, nickel and copper synergistically form the Cu-rich@Ni-rich@Ir-rich multielement alloy double-core-shell architecture, consistent with the linear scan of the elements (Fig. 2f). The crystal structure of the IrNiCu HCSA was analyzed by XRD. As shown in Fig. S2, the spectrum exhibits four main diffraction peaks matching the typical fcc crystalline structure of Ir (PDF \#06-0598), corresponding to the (111), (200), (220) and (311) crystal planes. Furthermore, the doping of $\mathrm{Ni}$ and $\mathrm{Cu}$ atoms with smaller radii results in a shift of the spectrum to a higher angle, and this shift reveals the formation of a well-defined $\mathrm{IrNiCu}$ structure. The EDX spectroscopy of the IrNiCu HCSA (Fig. S3) further attests to the coexistence of all the elements with the Ir weight percentage of $61.9 \%$, same as the initial loading percentage. According to the XPS results (Table S1), the percentage of Ir atoms in IrNiCu HCSA is $76.2 \%$, larger than that in EDS (33.9\%), indicating that there are more Ir atoms on the surface, consistent with the element mapping results.

To further analyze the ternary IrNiCu HCSA, two binary $\mathrm{IrNi}$ and $\mathrm{IrCu}$ NA were prepared for comparison. Fig. S4a shows a typical TEM image of an IrNi NA, composed of many small particles interlaced and stacked. The lattice spacing was measured to be between 0.216 and
$0.218 \mathrm{~nm}$ (Fig. S4b). Fig. S4c shows the element distribution of the IrNi NA. It can be seen that the Ni is concentrated in the center, while the Ir distributes in the periphery, indicating that the Ni first nucleates and then the Ir grows during the synthesis process. The element linear scan distribution (Fig. S4d) further proves the above conclusion. According to the particle size analysis in Fig. S5, the particle size of the IrNi NA is concentrated between 25 and $40 \mathrm{~nm}$, smaller than that of the $\mathrm{IrNiCu}$ HCSA. The XRD pattern (Fig. S6) shows that the four major peaks of the corresponding Ir are all offset to a high angle, indicating that the IrNi alloy is well formed with the addition of Ni. Fig. S7 shows that the Ir weight percentage is $76.2 \%$ in the IrNi NA. The morphology of the $\mathrm{IrCu}$ NA (Fig. S8a) is a membrane-like polyhedron structure, and the crystal plane spacing (Fig. S8b) is between 0.210 and $0.220 \mathrm{~nm}$, assigned to the (111) crystal plane. Fig. S8c shows that the element distribution of the $\mathrm{IrCu}$ is relatively uniform, with no obvious area of concentration, which is consistent with the linear scan element distribution (Fig. S8d). In addition, its particle size (Fig. S9) is smaller than that of the IrNi NA. The XRD results of the $\mathrm{IrCu}$ are similar to those of the IrNi NA (Fig. S10).

The electronic structures of the IrNiCu HCSA, IrNi NA and $\mathrm{IrCu}$ NA were investigated by XPS. The XPS survey scan spectra suggest the existence of iridium, nickel and copper in the samples (Fig. 3a). Fig. 3b depicts the core level spectra of the Ir $4 \mathrm{f}$ electrons of the three different samples. For IrNiCu HCSA, the Ir $4 \mathrm{f}_{5 / 2}$ and $\operatorname{Ir} 4 \mathrm{f}_{7 / 2}$ peaks 

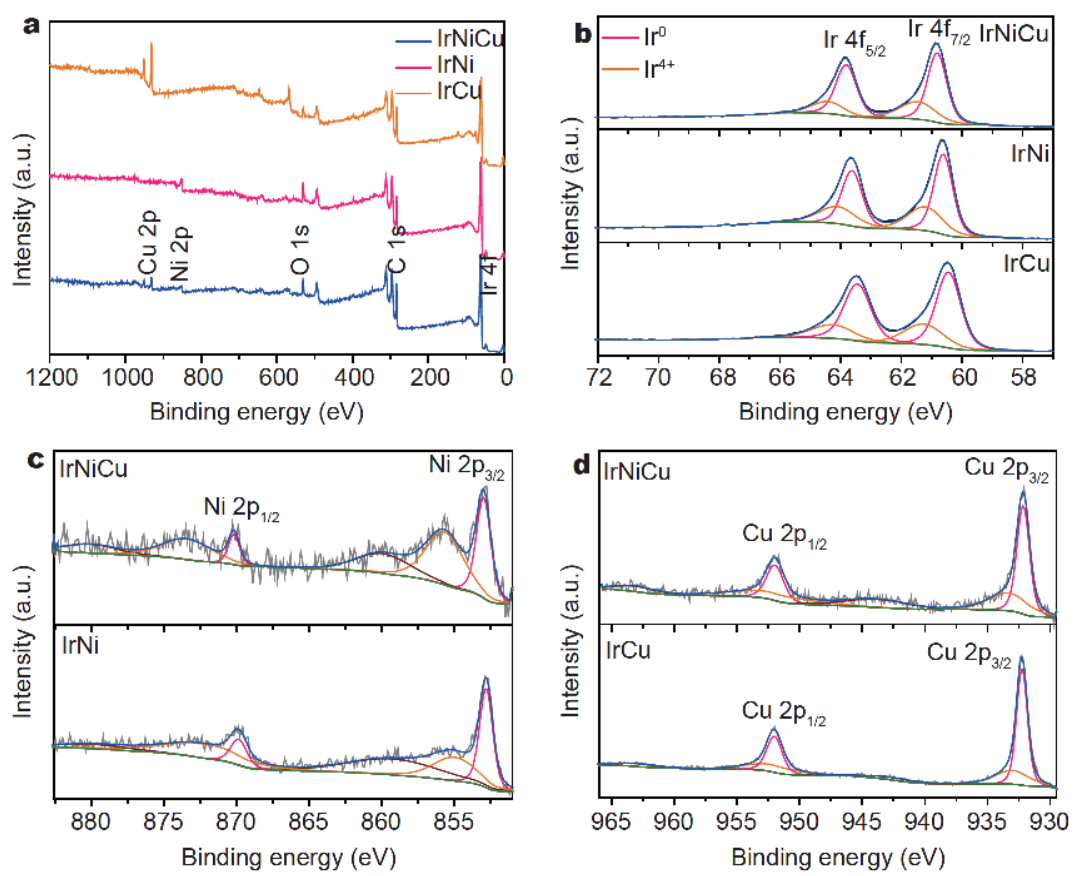

Figure 3 (a) XPS survey spectra, and core levels spectra of (b) Ir 4f, (c) Ni 2p, and (d) Cu 2p for the IrNiCu HCSA, IrNi NA and IrCu NA samples.

are deconvoluted into two sets of peaks centered at 63.8 and $60.8 \mathrm{eV}$ for the $\operatorname{Ir}^{0}$ and at 64.4 and $61.4 \mathrm{eV}$ for the $\mathrm{Ir}^{4+}$ $[47,48]$. The presence of iridium at a high chemical valence is due to its oxidation in the air. For IrNi NA and $\mathrm{IrCu} \mathrm{NA}$, their Ir $4 \mathrm{f}$ peaks show the same rule as that of IrNiCu HCSA, but there are slight differences. Specifically, the Ir $4 f_{5 / 2}$ and $\operatorname{Ir} 4 f_{7 / 2}$ peaks of the IrNi NA are both shifted by $0.2 \mathrm{eV}$ in the direction of low binding energy, while the $\mathrm{IrCu} \mathrm{NA}$ is shifted by $0.4 \mathrm{eV}$ in the same direction, meaning the Ir in IrNi NA and IrCu NA has a lower valence state. The deconvoluted $\mathrm{Ni} 2 \mathrm{p}$ is shown in Fig. 3c, the binding energies of 855.6 and $873.4 \mathrm{eV}$ can be attributed to $\mathrm{Ni}^{2+}\left(\mathrm{NiO}\right.$ or $\left.\mathrm{Ni}(\mathrm{OH})_{2}\right)$, and the peaks at 852.8 and $869.9 \mathrm{eV}$ correspond to $\mathrm{Ni}^{0}$ [49]. The same rule is applied to IrNi NA, except that all the corresponding peaks of IrNi NA are shifted by $0.2-0.6 \mathrm{eV}$ in the direction of low binding energy. Fig. $3 \mathrm{~d}$ shows that the binding energy of $\mathrm{Cu} 2 \mathrm{p}_{3 / 2}$ of IrNiCu HCSA shifts to a lower value $(932.1 \mathrm{eV})$ compared with standard $\mathrm{Cu} 2 \mathrm{p}_{3 / 2}(933 \mathrm{eV})$, while the binding energy of $\mathrm{Cu} 2 \mathrm{p}_{3 / 2}$ in $\mathrm{IrCu}$ NA decreases slightly $(932.3 \mathrm{eV})$, indicating that the partial charge transfer from $\mathrm{Cu}$ to Ir results in a lower d-band center of Ir, improving its electrochemical properties [50]. The peaks at 952.0 and $952.3 \mathrm{eV}$ represent the $2 \mathrm{p}_{1 / 2}$ peaks of $\mathrm{Cu}$ in $\mathrm{IrNiCu}$ HCSA and IrCu NA, respectively. The above four peaks represent the zero valence state of copper in the samples $[51,52] . \mathrm{Cu}^{2+}$ still exists in the samples evidenced by the corresponding binding energy $(\sim 933.5 \mathrm{eV})$ [53].

The HER electrocatalytic properties of the $\mathrm{IrNiCu}$ HCSA were evaluated in $0.5 \mathrm{~mol} \mathrm{~L}^{-1} \mathrm{H}_{2} \mathrm{SO}_{4}$ solution. The polarization curves of HER indicate that the overpotentials of $\mathrm{IrNiCu} \mathrm{HCSA}$, IrNi NA, IrCu NA and Pt/C are $41,55,70$ and $39 \mathrm{mV}$, respectively, at a current density of $10 \mathrm{~mA} \mathrm{~cm}^{-2}$ (Fig. 4a), revealing the superior catalytic activity of the IrNiCu HCSA compared with the $\mathrm{Pt} / \mathrm{C}$. The corresponding Tafel plots are illustrated in Fig. $4 \mathrm{~b}$, where the IrNiCu HCSA shows the lowest Tafel slope $(21.4 \mathrm{mV} / \mathrm{dec})$ and is almost the same as the commercial $\mathrm{Pt} / \mathrm{C}(21.0 \mathrm{mV} / \mathrm{dec})$. The HER kinetics of the catalysts at the electrode/electrolyte interface were further established by EIS measurements at $-0.05 \mathrm{~V}$ (vs. RHE) (Fig. 4c). The Pt/C and IrNiCu HCSA exhibit the smallest resistance, possessing a lower charge-transfer resistance and faster charge transfer rate [19]. CV curves under different scanning rates $(5,10,20,40,60,80$ and $100 \mathrm{mV} \mathrm{s}^{-1}$ ) in the voltage range of $0.9-1.0 \mathrm{~V}$ (vs. RHE) were performed as shown in Figs S11-S15. Under the same voltage, the current density of the IrNiCu HCSA is the maximum value. Meanwhile, the ECSA was estimated by the $C_{\mathrm{dl}}$ using a CV measurement in Fig. S16. The $C_{\mathrm{dl}}$ of IrNiCu HCSA is $71.19 \mathrm{mF} \mathrm{cm}^{-2}$, much larger than those of $\mathrm{IrNi} \mathrm{NA}\left(41.24 \mathrm{mF} \mathrm{cm}^{-2}\right)$ and $\operatorname{IrCu} \mathrm{NA}$ $\left(22.64 \mathrm{mF} \mathrm{cm}^{-2}\right)$, indicating that the hierarchical structure 

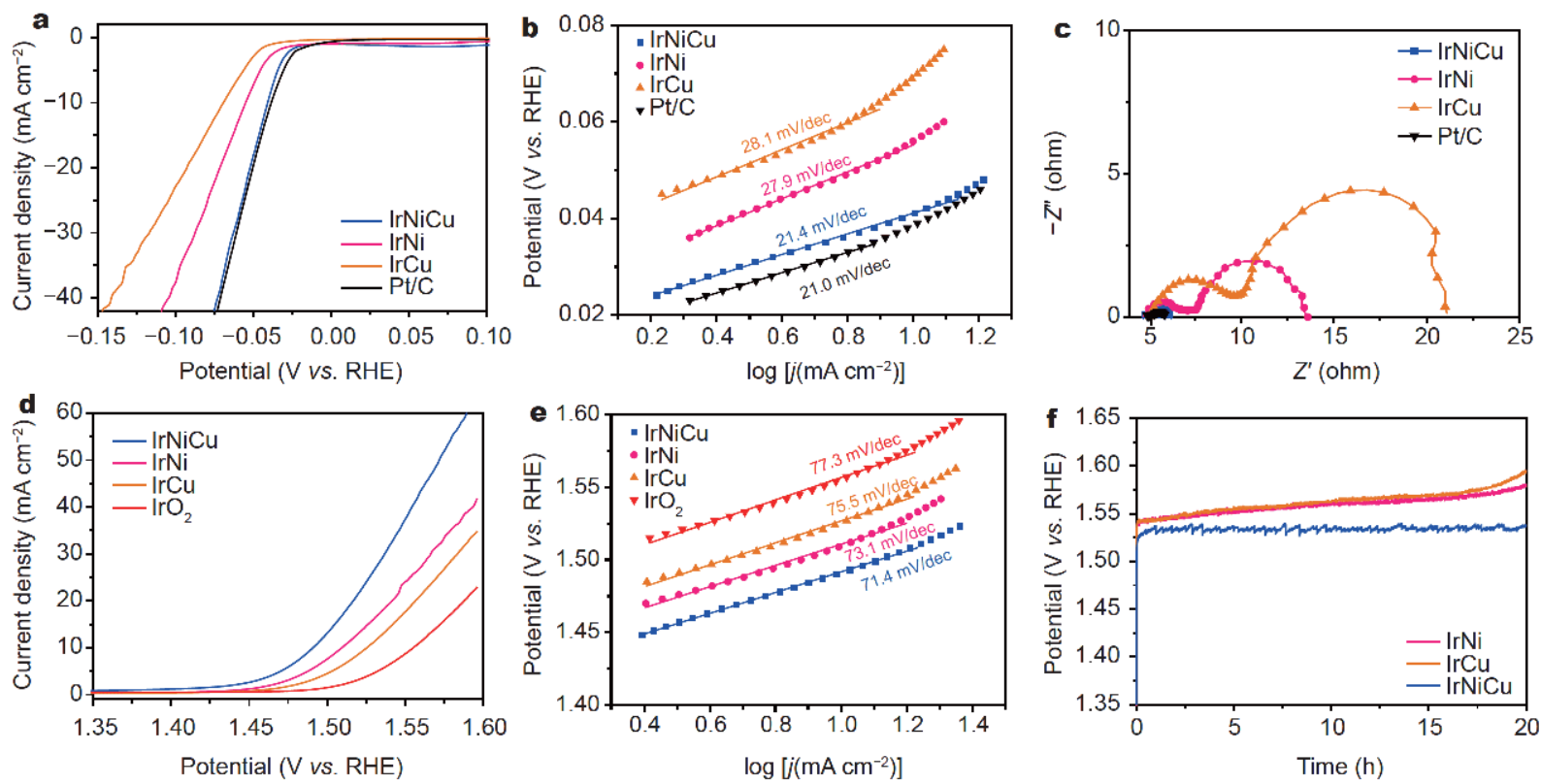

Figure 4 (a, d) LSV curves for HER and OER performance of IrNiCu HCSA, IrNi NA, IrCu NA, Pt/C and $\operatorname{IrO}_{2}$ in $\mathrm{N}_{2}$-saturated 0.5 mol L $\mathrm{L}^{-1} \mathrm{H}_{2} \mathrm{SO}_{4}$ solution at the potential scan rate of $5 \mathrm{mV} \mathrm{s}^{-1}$, (b) Tafel plots and (c) EIS of IrNiCu HCSA, IrNi NA, IrCu NA and Pt/C for HER at - $0.05 \mathrm{~V}$ ( $v s$. RHE). (e) Tafel plots and (f) chronopotentiometry curve of IrNiCu HCSA, IrNi NA and IrCu NA in $0.5 \mathrm{~mol} \mathrm{~L}^{-1} \mathrm{H}_{2} \mathrm{SO}_{4}$ at a constant current density of $10 \mathrm{~mA} \mathrm{~cm}^{-2}$.

offers vast active sites for catalytic performance improvement.

The OER activity of the IrNiCu HCSA was also investigated in $0.5 \mathrm{~mol} \mathrm{~L}^{-1} \mathrm{H}_{2} \mathrm{SO}_{4}$, as illustrated in Fig. $4 \mathrm{~d}$. The IrNiCu HCSA exhibits the lowest overpotential $(262 \mathrm{mV})$, and the $\mathrm{IrCu}$ NA shows the highest overpotential $(296 \mathrm{mV})$ at a current density of $10 \mathrm{~mA} \mathrm{~cm}^{-2}$. In addition, the electrochemical performance of the $\mathrm{IrNiCu}$ HCSA, IrNi NA and IrCu NA are all better than that of commercial $\mathrm{IrO}_{2}(326 \mathrm{mV})$. Fig. S17 shows the overpotentials of the various specimens at different current densities. It can be seen that the corresponding overpotential of $\mathrm{IrNiCu} \mathrm{HCSA}$ is the lowest at 1,10 and $20 \mathrm{~mA} \mathrm{~cm}^{-2}$, showing the best catalytic property for OER. The $\mathrm{IrNiCu}$ HCSA exhibits the lowest Tafel slope of $71.4 \mathrm{mV} / \mathrm{dec}$ (Fig. 4e), lower than that of $\mathrm{IrO}_{2}$ $(77.3 \mathrm{mV} / \mathrm{dec})$, confirming its impressive OER electrocatalytic activity. The EIS results at $1.5 \mathrm{~V}$ ( $v s$. RHE), as shown in Fig. S18, demonstrate that the IrNiCu HCSA displays the smallest impedance and lowest charge transfer resistance compared with the IrNi NA, $\mathrm{IrCu} \mathrm{NA}$ and $\mathrm{IrO}_{2}$. Durability is an important parameter to evaluate the catalytic performance. The stability of the catalysts was investigated by a chronopotentiometry measurement at a constant current density of $10 \mathrm{~mA} \mathrm{~cm}^{-2}$ in $0.5 \mathrm{~mol} \mathrm{~L}^{-1} \mathrm{H}_{2} \mathrm{SO}_{4}$ solution (Fig. $4 \mathrm{f}$ ). After a con- tinuous $20 \mathrm{~h}$ test, the voltage of IrNiCu HCSA increases by only $2 \%$ compared with the initial one, while that of $\mathrm{IrCu} \mathrm{NA}$ increases by $5 \%$, indicating the excellent durability of IrNiCu HCSA in acidic solution. We also investigated the TEM of the IrNiCu HCSA, IrNi NA and IrCu NA catalysts after the OER stability test (Figs S19S21), indicating that the morphology had no obvious change. The corresponding EDX (Table S2) shows that its composition has not significantly changed, indicating the morphology and composition remained.

To investigate the possibility of simultaneously employing the IrNiCu HCSA as an efficient bifunctional catalyst for both HER and OER, an electrode of the catalysts coated on carbon paper $(1 \mathrm{~cm} \times 1 \mathrm{~cm})$ was fabricated and tested as both the anode and cathode in a two-electrode system in acidic solution (Fig. 5d). The electrolysis that applied the IrNiCu HCSA as bifunctional catalysts exhibits exceptional performance for overall water splitting. When the current density was $10 \mathrm{~mA} \mathrm{~cm}$, the voltages of $\mathrm{IrNiCu} \mathrm{HCSA}$, IrNi NA and IrCu NA were $1.56,1.62$ and $1.65 \mathrm{~V}$, respectively (Fig. 5a). The stability of the IrNiCu HCSA for overall water splitting was evaluated by a chronopotentiometry measurement at a constant current density of $10 \mathrm{~mA} \mathrm{~cm}{ }^{-2}$ in $0.5 \mathrm{~mol} \mathrm{~L}^{-1}$ $\mathrm{H}_{2} \mathrm{SO}_{4}$ solution (Fig. 5b). The IrNiCu HCSA has the lowest voltage, at $\sim 1.53 \mathrm{~V}$, and after $20 \mathrm{~h}$ of continuous 

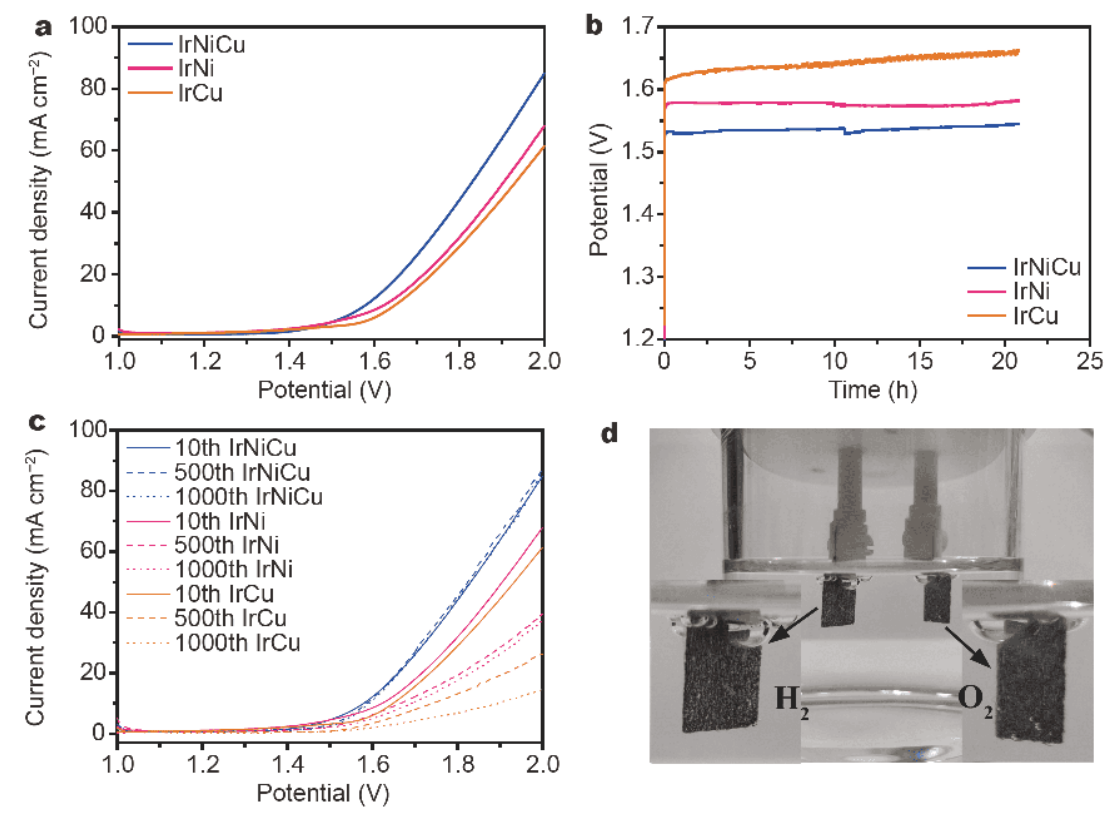

Figure 5 Overall-water-splitting performance of IrNiCu HCSA, IrNi NA and IrCu NA. (a) Polarization curves of IrNiCu HCSA, IrNi NA and IrCu $\mathrm{NA}$ in $0.5 \mathrm{~mol} \mathrm{~L}^{-1} \mathrm{H}_{2} \mathrm{SO}_{4}$ solution at a scan rate of $5 \mathrm{mV} \mathrm{s}^{-1}$. (b) Chronopotentiometry curve of IrNiCu HCSA, IrNi NA and IrCu NA in $0.5 \mathrm{~mol} \mathrm{~L}{ }^{-1}$ $\mathrm{H}_{2} \mathrm{SO}_{4}$ at a constant current density of $10 \mathrm{~mA} \mathrm{~cm}^{-2}$. (c) Polarization curves of IrNiCu HCSA, IrNi NA and IrCu NA for overall water splitting after 10, 500, and 1000 cycles. (d) Optical image of electrocatalytic water electrolysis.

catalysis, the voltage increases by only $0.8 \%$, demonstrating the excellent stability of IrNiCu HCSA. A homemade exhaust device was set up to collect the gas products $\left(\mathrm{H}_{2}\right.$ and $\left.\mathrm{O}_{2}\right)$ to detect the catalytic efficiency of IrNiCu HSCA for overall water splitting in Fig. S22. The faradaic efficiencies of IrNiCu HCSA are almost 100\% for both HER and OER. We further evaluated the long-term stability of IrNiCu HCSA, IrNi NA and IrCu NA toward water splitting by cycling the potential between 1.0 and $2.0 \mathrm{~V}$ for 10,500 and 1000 cycles, respectively. It can be clearly seen from Fig. $5 \mathrm{c}$ that IrNiCu HCSA has the best stability, and the polarization curve basically does not change even after 1000 cycles, compared with the serious changes observed in that of the IrNi and IrCu NA.

\section{CONCLUSIONS}

In summary, we demonstrated a simple, one-step method for the synthesis of IrNiCu nanoframes with hierarchical features and double core-shell structures assisted by oil bath reactions and applied it as an efficient bifunctional catalyst for overall water splitting catalysis in acidic environments. By comparing various Ir-based alloy specimens, we found that the IrNiCu HCSA exhibited excellent performance with an OER activity superior to that of $\mathrm{IrO}_{2}$ (overpotential of $262 \mathrm{mV}$ and Tafel slope of $71.4 \mathrm{mV} / \mathrm{dec} v s .326 \mathrm{mV}$ and $77.3 \mathrm{mV} / \mathrm{dec}$ at a current density of $10 \mathrm{~mA} \mathrm{~cm}^{-2}$ ) and a HER activity close to that of $\mathrm{Pt} / \mathrm{C} \quad(41 \mathrm{mV}$ and $21.4 \mathrm{mV} / \mathrm{dec}$ vs. $39 \mathrm{mV}$ and $21.0 \mathrm{mV} / \mathrm{dec}$ ). An outstanding overall water splitting performance was also observed with a current density that reached $10 \mathrm{~mA} \mathrm{~cm}^{-2}$ at a potential of only $1.53 \mathrm{~V}$ and remained stable for a long time. The exceptional electrochemical performances are mainly attributed to the large electrochemical active areas brought by the hierarchical nanoframe architecture and the synergistic effect from the three elements of $\mathrm{Ir}, \mathrm{Ni}$ and $\mathrm{Cu}$ and the double core-shell structure. This work illustrates a novel strategy to design Ir-based multimetallic HCSA as a bifunctional catalyst with remarkable electrocatalytic activity and robust durability for water splitting in an acidic solution and provides a very broad prospect for practical application in a PEMWE.

\section{Received 4 July 2019; accepted 2 September 2019;}

published online 26 September 2019

1 Xing $\mathrm{Z}, \mathrm{Wu} \mathrm{H}, \mathrm{Wu} \mathrm{L}$, et al. A multifunctional vanadium-doped cobalt oxide layer on silicon photoanodes for efficient and stable photoelectrochemical water oxidation. J Mater Chem A, 2018, 6: 21167-21177

2 Wang C, Sui Y, Xiao G, et al. Synthesis of $\mathrm{Cu}-\mathrm{Ir}$ nanocages with enhanced electrocatalytic activity for the oxygen evolution reaction. J Mater Chem A, 2015, 3: 19669-19673

3 Shan J, Ling T, Davey K, et al. Transition-metal-doped RuIr bi- 
functional nanocrystals for overall water splitting in acidic environments. Adv Mater, 2019, 31: 1900510

4 Lv F, Feng J, Wang $\mathrm{K}$, et al. Iridium-tungsten alloy nanodendrites as $\mathrm{pH}$-universal water-splitting electrocatalysts. ACS Cent Sci, 2018, 4: 1244-1252

5 Zhu X, Tang C, Wang HF, et al. Monolithic-structured ternary hydroxides as freestanding bifunctional electrocatalysts for overall water splitting. J Mater Chem A, 2016, 4: 7245-7250

6 Ou G, Fan P, Zhang H, et al. Large-scale hierarchical oxide nanostructures for high-performance electrocatalytic water splitting. Nano Energy, 2017, 35: 207-214

7 Chen Y, Xu S, Zhu S, et al. Millisecond synthesis of CoS nanoparticles for highly efficient overall water splitting. Nano Res, 2019, 12: $2259-2267$

8 Luo M, Cai Z, Wang C, et al. Phosphorus oxoanion-intercalated layered double hydroxides for high-performance oxygen evolution. Nano Res, 2017, 10: 1732-1739

$9 \mathrm{Bu} \mathrm{F}$, Chen W, Gu J, et al. Microwave-assisted CVD-like synthesis of dispersed monolayer/few-layer N-doped graphene encapsulated metal nanocrystals for efficient electrocatalytic oxygen evolution. Chem Sci, 2018, 9: 7009-7016

10 Wang X, Vasileff A, Jiao Y, et al. Electronic and structural engineering of carbon-based metal-free electrocatalysts for water splitting. Adv Mater, 2019, 31: 1803625

11 Hao S, Chen L, Yu C, et al. NiCoMo hydroxide nanosheet arrays synthesized via chloride corrosion for overall water splitting. ACS Energy Lett, 2019, 4: 952-959

12 Xue Q, Gao W, Zhu J, et al. Carbon nanobowls supported ultrafine iridium nanocrystals: An active and stable electrocatalyst for the oxygen evolution reaction in acidic media. J Colloid Interface Sci, 2018, 529: 325-331

13 Duan $\mathrm{H}$, Li D, Tang $\mathrm{Y}$, et al. High-performance $\mathrm{Rh}_{2} \mathrm{P}$ electrocatalyst for efficient water splitting. J Am Chem Soc, 2017, 139: 5494-5502

14 Reier T, Nong HN, Teschner D, et al. Electrocatalytic oxygen evolution reaction in acidic environments-reaction mechanisms and catalysts. Adv Energy Mater, 2017, 7: 1601275

15 Liu X, Wen B, Guo R, et al. A porous nickel cyclotetraphosphate nanosheet as a new acid-stable electrocatalyst for efficient hydrogen evolution. Nanoscale, 2018, 10: 9856-9861

16 Lu S, Zhuang Z. Electrocatalysts for hydrogen oxidation and evolution reactions. Sci China Mater, 2016, 59: 217-238

$17 \mathrm{Wu}$ S, Zhu Y, Huo Y, et al. Bimetallic organic frameworks derived $\mathrm{CuNi} /$ carbon nanocomposites as efficient electrocatalysts for oxygen reduction reaction. Sci China Mater, 2017, 60: 654-663

18 Feng J, Lv F, Zhang W, et al. Iridium-based multimetallic porous hollow nanocrystals for efficient overall-water-splitting catalysis. Adv Mater, 2017, 29: 1703798

19 Jiang P, Chen J, Wang C, et al. Tuning the activity of carbon for electrocatalytic hydrogen evolution via an iridium-cobalt alloy core encapsulated in nitrogen-doped carbon cages. Adv Mater, 2018, 30: 1705324

20 Fu L, Yang F, Cheng G, et al. Ultrathin Ir nanowires as highperformance electrocatalysts for efficient water splitting in acidic media. Nanoscale, 2018, 10: 1892-1897

21 Han N, Yang KR, Lu Z, et al. Nitrogen-doped tungsten carbide nanoarray as an efficient bifunctional electrocatalyst for water splitting in acid. Nat Commun, 2018, 9: 924-934

22 Yin J, Fan Q, Li Y, et al. Ni-C-N nanosheets as catalyst for hydrogen evolution reaction. J Am Chem Soc, 2016, 138: 14546-
14549

23 Pi Y, Zhang N, Guo S, et al. Ultrathin laminar Ir superstructure as highly efficient oxygen evolution electrocatalyst in broad $\mathrm{pH}$ range. Nano Lett, 2016, 16: 4424-4430

24 Zaman WQ, Sun W, Tariq M, et al. Iridium substitution in nickel cobaltite renders high mass specific OER activity and durability in acidic media. Appl Catal B-Environ, 2019, 244: 295-302

25 Zhang $\mathrm{Y}, \mathrm{Wu} \mathrm{C}$, Jiang $\mathrm{H}$, et al. Atomic iridium incorporated in cobalt hydroxide for efficient oxygen evolution catalysis in neutral electrolyte. Adv Mater, 2018, 30: 1707522

26 Shi Q, Zhu C, Zhong $\mathrm{H}$, et al. Nanovoid Incorporated $\mathrm{Ir}_{x} \mathrm{Cu}$ metallic aerogels for oxygen evolution reaction catalysis. ACS Energy Lett, 2018, 3: 2038-2044

27 Li D, Zong Z, Tang Z, et al. Total water splitting catalyzed by Co@Ir core-shell nanoparticles encapsulated in nitrogen-doped porous carbon derived from metal-organic frameworks. ACS Sustain Chem Eng, 2018, 6: 5105-5114

28 Pi Y, Shao Q, Wang P, et al. General formation of monodisperse IrM $(\mathrm{M}=\mathrm{Ni}, \mathrm{Co}, \mathrm{Fe})$ bimetallic nanoclusters as bifunctional electrocatalysts for acidic overall water splitting. Adv Funct Mater, 2017, 27: 1700886

29 Won JE, Kwak DH, Han SB, et al. PtIr/ $/ \mathrm{Ti}_{4} \mathrm{O}_{7}$ as a bifunctional electrocatalyst for improved oxygen reduction and oxygen evolution reactions. J Catal, 2018, 358: 287-294

30 Liu B, Wang C, Chen Y. Surface determination and electrochemical behavior of $\mathrm{IrO}_{2}-\mathrm{RuO}_{2}-\mathrm{SiO}_{2}$ ternary oxide coatings in oxygen evolution reaction application. Electrochim Acta, 2018, 264: $350-357$

31 Lv H, Zhang G, Hao C, et al. Activity of $\mathrm{IrO}_{2}$ supported on tantalum-doped $\mathrm{TiO}_{2}$ electrocatalyst for solid polymer electrolyte water electrolyzer. RSC Adv, 2017, 7: 40427-40436

32 Fu L, Cheng G, Luo W. Colloidal synthesis of monodisperse trimetallic IrNiFe nanoparticles as highly active bifunctional electrocatalysts for acidic overall water splitting. J Mater Chem A, 2017, 5: 24836-24841

33 Yang W, Wang Z, Zhang W, et al. Electronic-structure tuning of water-splitting nanocatalysts. Trends Chem, 2019, 1: 259-271

34 Wang D, Peng Q, Li Y. Nanocrystalline intermetallics and alloys. Nano Res, 2010, 3: 574-580

35 Park J, Sa YJ, Baik H, et al. Iridium-based multimetallic nanoframe@nanoframe structure: An efficient and robust electrocatalyst toward oxygen evolution reaction. ACS Nano, 2017, 11: 5500-5509

36 Tackett BM, Sheng W, Kattel S, et al. Reducing iridium loading in oxygen evolution reaction electrocatalysts using core-shell particles with nitride cores. ACS Catal, 2018, 8: 2615-2621

37 Nong HN, Oh HS, Reier T, et al. Oxide-supported $\mathrm{IrNiO}_{x}$ coreshell particles as efficient, cost-effective, and stable catalysts for electrochemical water splitting. Angew Chem Int Ed, 2015, 54: 2975-2979

38 Chen $\mathrm{Z}$, Wu R, Liu $\mathrm{Y}$, et al. Ultrafine Co nanoparticles encapsulated in carbon-nanotubes-grafted graphene sheets as advanced electrocatalysts for the hydrogen evolution reaction. Adv Mater, 2018, 30: 1802011

39 Luo Z, Tan C, Lai Z, et al. A simple electrochemical method for conversion of Pt wires to Pt concave icosahedra and nanocubes on carbon paper for electrocatalytic hydrogen evolution. Sci China Mater, 2018, 62: 115-121

40 Zheng X, Cao Y, Han X, et al. Pt embedded $\mathrm{Ni}_{3} \mathrm{Se}_{2} @ \mathrm{NiOOH}$ coreshell dendrite-like nanoarrays on nickel as bifunctional electro- 
catalysts for overall water splitting. Sci China Mater, 2019, 62: 1096-1104

41 Wang Q, Dou X, Chen X, et al. Reevaluating protein photoluminescence: Remarkable visible luminescence upon concentration and insight into the emission mechanism. Angew Chem Int Ed, 2019, 58: 12667-12673

42 Zheng S, Fang F, Zhou G, et al. Hydrogen storage properties of space-confined $\mathrm{NaAlH}_{4}$ nanoparticles in ordered mesoporous silica. Chem Mater, 2008, 20: 3954-3958

43 Zhang N, Guo S, Zhu X, et al. Hierarchical $\mathrm{Pt} / \mathrm{Pt}_{x} \mathrm{~Pb}$ core/shell nanowires as efficient catalysts for electrooxidation of liquid fuels. Chem Mater, 2016, 28: 4447-4452

44 Feng $\mathrm{Y}$, Shao Q, Huang B, et al. Surface engineering at the interface of core/shell nanoparticles promotes hydrogen peroxide generation. Natl Sci Rev, 2018, 5: 895-906

45 Wang Q, Zhu Y, Xue J, et al. General synthesis of porous mixed metal oxide hollow spheres with enhanced supercapacitive properties. ACS Appl Mater Interfaces, 2016, 8: 17226-17232

46 Zhao J, He Y, Chen Z, et al. Engineering the surface metal active sites of nickel cobalt oxide nanoplates toward enhanced oxygen electrocatalysis for Zn-air battery. ACS Appl Mater Interfaces, 2019, 11: 4915-4921

47 Yuan L, Yan Z, Jiang L, et al. Gold-iridium bifunctional electrocatalyst for oxygen reduction and oxygen evolution reactions. J Energy Chem, 2016, 25: 805-810

48 Shim JH, Kim JE, Cho YB, et al. Oxidation-state dependent electrocatalytic activity of iridium nanoparticles supported on graphene nanosheets. Phys Chem Chem Phys, 2013, 15: 15365

49 Teng X, Wang J, Ji L, et al. Fabrication of three-dimensional multiscale porous alloy foams at a planar substrate for efficient water splitting. ACS Sustain Chem Eng, 2019, 7: 5412-5419

50 Jiang K, Cai WB. Carbon supported Pd-Pt-Cu nanocatalysts for formic acid electrooxidation: Synthetic screening and componential functions. Appl Catal B-Environ, 2014, 147: 185-192

51 Zhu Y, Chen G, Zhong Y, et al. A surface-modified antiperovskite as an electrocatalyst for water oxidation. Nat Commun, 2018, 9: 2326

52 Sheng J, Kang J, Ye H, et al. Porous octahedral PdCu nanocages as highly efficient electrocatalysts for the methanol oxidation reaction. J Mater Chem A, 2018, 6: 3906-3912

53 Biesinger MC, Lau LWM, Gerson AR, et al. Resolving surface chemical states in XPS analysis of first row transition metals, oxides and hydroxides: Sc, Ti, V, Cu and Zn. Appl Surf Sci, 2010, 257: $887-898$

Acknowledgements This work was supported by the National Natural Science Foundation of China (51571151, 51701139, 51671143 and U1601216).

Author contributions Zhang J, Chen Y, Rao D and Deng Y conceived the idea and designed the experiment. Zhang J, Chen Z, Liu C and Nie A carried out the synthesis, characterization and electrochemical experiments. All authors participated in the data analysis and contributed to the manuscript writing.

Conflict of interest The authors declare no competing financial interest.
Supplementary information Supplementary data including TEM images, EDS pattern, XRD pattern and supplementary electrochemical properties data are available in the online version of the paper.

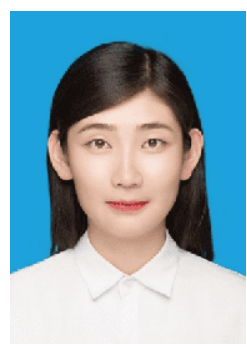

Jie Zhang obtained her Bachelor's degree from the School of Materials Science and Engineering, Chang'an University in 2017. She is now a graduate student in the School of Materials Science and Engineering, Tianjin University. Her main research focuses on the synthesis and characterization of iridium nanomaterials for electrocatalysis.

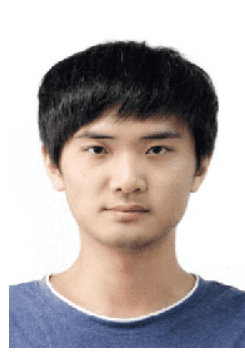

Zelin Chen received his Bachelor and Master degrees from Nanjing Tech University in 2012 and 2015, respectively. Now, he is a PhD candidate majored in material science under the supervision of Prof. Wenbin $\mathrm{Hu}$ and Prof. Yida Deng at Tianjin University. His research interests focus on the design and controllable synthesis of noble-metal-based nanomaterials and their electrocatalytic applications in fuel cells.

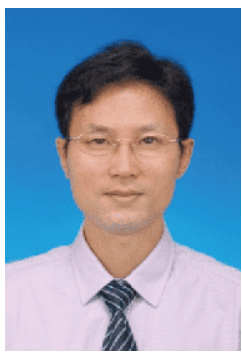

Yida Deng is a Professor in the School of Materials Science and Engineering, Tianjin University. He received his $\mathrm{PhD}$ from Shanghai Jiao Tong University in 2006. His research interests include the metal and metal oxide nanostructures for electrochemical and energy applications.

\section{分级双核壳架构铱基多金属合金催化剂的高效全 解水研究}

张洁 ${ }^{1}$, 陈泽霖 ${ }^{1}$, 刘畅 ${ }^{1}$, 赵君 ${ }^{1}$, 刘思郬 ${ }^{1}$, 饶德伟 ${ }^{2}$, 聂安民 ${ }^{3}$, 陈亚楠 ${ }^{1^{*}}$, 邓意达 ${ }^{1^{*}}$, 胡文涁 ${ }^{1,4}$

摘要 电解水制氢是解决环境和能源危机的一种有效策略. 本文采 用简便的一步油浴法合成分级双核壳架构(HCSA)铱基多金属合金 催化剂, 并将其应用于酸性环境中的全解水. 所制备的 $\mathrm{IrNiCu}$ $\mathrm{HCSA}$ 表现出可以与 $\mathrm{Pt} / \mathrm{C}$ 相媲美的氢析出(HER)性能和优于 $\mathrm{IrO}_{2}$ 的 氧析出(OER)性能. IrNiCu HCSA作为一种双功能催化剂也显示出 卓越的全解水性能, 在酸性环境中当电流密度为 $10 \mathrm{~mA} \mathrm{~cm}^{-2}$ 时电 压仅为 $1.53 \mathrm{~V}$, 并且在持续催化 $20 \mathrm{~h}$ 后性能基本不变, 具有很好的 稳定性. 本研究为新型铱基多金属合金结构的设计与合成奠定了 基础, 有望应用于电化学催化水分解领域. 